\title{
Effect of olive oil and freeze dried beetroot powder on quality characteristics of chicken meat patties by response surface methodology study
}

\author{
- RAYEES Ul ISLAM AND MOHAMMAD ALI KHAN* \\ Department of Post Harvest Engineering and Technology, Aligarh Muslim University, ALIGARH (U.P.) INDIA \\ Email : billuraiz@gmail.com; makamu4@yahoo.co.in
}

\section{SUMMARY:}

A two factor Central Composite Design (CCD) was applied to study the major effects and interactions of processing variables such as olive oil (5-15\%) and beetroot powder (1-5\%) on cooking properties of chicken patties. The addition of olive oil increased reduction in diameter, shrinkage and emulsion stability values however, reduction in thickness, fat and moisture retention, cooking yield values decreased as the amount of olive oil increased. On the other hand, the incorporation of beetroot powder decreased reduction in thickness and diameter, shrinkage and emulsion stability. Cooking yield and fat and moisture retention values were found to increase by the addition of beetroot powder.

KEY WORDS : Beetroot powder, Olive oil, Patty, Response surface methodology, Cooking properties

How to cite this paper : Islam, Rayees Ul and Khan, Mohammad Ali (2016). Effect of olive oil and freeze dried beetroot powder on quality characteristics of chicken meat patties by response surface methodology study. Internat. J. Proc. \& Post Harvest Technol., 7 (2) : 220-227. DOI: 10.15740/HAS/IJPPHT/7.2/220-227. 